\title{
Design and Experimental Validation of a Stable Two Stage Estimator for Automotive Sideslip Angle and Tire Parameters
}

\author{
Frank Naets, Sebastiaan van Aalst, Boulaid Boulkroune, Norddin El Ghouti and Wim Desmet
}

\begin{abstract}
This work proposes and experimentally validates a two-stage approach for coupled lateral vehicle state and tire model estimation. In a first stage an extended Kalman filter is employed which provides vehicle slip angles and lateral tire forces from commercial low cost vehicle sensors. The obtained estimates are exploited in the second stage where a (quasi-static) tire model is fitted to this data. A major issue in this estimation process is the typical instability of these estimators for situations with (prolonged) straight driving. This issue is traced back to a lack of local observability. The use of a variable model covariance is introduced as a practical method to obtain a stable estimator, irrespective of the unobservability. The developed methodology has a low computational load and the Kalman estimator is able to run in real-time, whereas the tire model parameter fitting is cheap enough to run online. The proposed methodology is validated experimentally and provides reliable results in variable driving conditions.
\end{abstract}

Index Terms-Sideslip angle, tire model, Kalman filter, stable estimation.

\section{INTRODUCTION}

Over the past decades driver assistance systems and (semi)active safety systems have become a standard in automotive industry [1]. Of paramount importance for the most optimal performance in a vehicle is an accurate knowledge of the system state and parameters. However, many of these variables cannot be measured directly in commercial vehicles. Especially for lateral dynamics control, variables like the the vehicle sideslip angle and wheel forces play a crucial role. This information could also yield improvements in the accuracy of positioning systems based on low-cost GPS sensors. The direct measurement of these variables in an accurate fashion requires very costly equipment, respectively optical speed sensors and force measuring wheels [2] which are not feasible for commercial vehicles. Moreover, besides the current state information on the vehicle, the knowledge of several parameters can be very valuable for predictive vehicle control such as model predictive path tracking. Of particular interest are tire parameters. In current practice, tire parameters are typically obtained from extensive offline testing [3], [4] . This is a very thorough

Copyright (c) 2015 IEEE. Personal use of this material is permitted. However, permission to use this material for any other purposes must be obtained from the IEEE by sending a request to pubs-permissions @ieee.org.

F. Naets and W. Desmet are with the Department of Mechanical Engineering, KU Leuven, 3001 Heverlee, Belgium, and Members of Flanders Make, e-mail: frank.naets@kuleuven.be

S. van Aalst, B. Boulkroune and N. El Ghouti are with Flanders Make, 3920 Lommel, Belgium

Manuscript received April 19, 2005; revised August 26, 2015. procedure to compare different tires and obtain detailed models which can be used for vehicle design. However, during the life cycle of a vehicle, these predetermined parameters have limited value as road conditions and tire wear have a huge impact. For control actions an accurate online knowledge of the tire properties can be a major advantage.

This work focuses on a two stage approach which enables real-time sideslip angle and lateral tire force estimation and online tire parameter estimation from standard vehicle sensors. These quantities are of paramount importance for the analysis and control of the lateral vehicle dynamics.

A range of estimators schemes have been proposed over the years for estimating different variables associated with lateral automotive dynamics. Many works have focused on determining the vehicle sideslip angle through a model based estimator. Initially methods employing dynamic vehicle models assumed a known tire model [5], [6]. However, these approaches have clear limitations for real-world applications where the tire parameters are not known accurately and behave nonlinearly which leads to considerable bias. More recently Ryu et al. [7] proposed a purely kinematic approach where the sideslip angle information can be obtained through the use of a 2-antenna GPS sensor rather than through the use of a dynamic model. This approach can be extended in order to also estimate tire parameters besides the sideslip angle [8]. Other alternative sensors, like the steering torque sensor present for electrical power assisted steering systems can be exploited as well for vehicle estimation [9]. Ray [10], [11] proposed the combined sideslip angle and tire parameter estimation through an extended Kalman filter approach. In this approach a joint estimator is employed to directly estimate the different tire forces along with the states of the model. Anderson et al. [12], [13] employs two estimators (a kinematic and a dynamic one) which are switched in order to provide stable behavior of the estimator when little lateral excitation is present. If only tire force estimation is of interest, the model can be reformulated in order to not require vehicle speed information, but in this case no information on the sideslip angle can be retrieved [14]. A proven stable sideslip angle and friction estimator is proposed by Grip et al. [15], but this approach cannot be directly extended to full tire parameter estimation. Baffet et al. [16], [17] proposed a coupled estimator for the sideslip angle and tire parameters. An adaptive linear model is employed for the tires which allows for adjustment in the case where the tire behaves nonlinear. More recently the focus has been on the development of methods with new sensor information which 
can be extracted from additional GPS units [18], from electric vehicles [19], [20] or from vehicles equipped with optical camera sensors [21]. However, obviously these approaches lack the general applicability of the aforementioned methods and the approach proposed in this paper. Also more advanced estimation schemes based on multi-model techniques have been proposed in recent literature [19], [22]. Davoodabadi et al. [23] have proposed a two-step estimation approach as well where a joint Kalman filter (or dual Kalman filter) is used to estimate both states and model parameters. However, estimator stability is not investigated and only simulated results are presented in this work. Lee et al. [24] have presented an approach for tire parameter estimation with faster convergence, but this approach is only applicable for linear tire behavior.

This paper proposes a two-step approach where a standard extended Kalman filter (EKF) approach [25] is used to jointly estimate the vehicle states and tire forces or stiffnesses and a second stage nonlinear fitting is performed for the nonlinear tire parameters. The first stage EKF can be replaced by alternative nonlinear state estimators, like the unscented Kalman filter or the particle filter [25]. In this work the EKF is chosen due to its low computational load, enabling real-time operation, and flexibility with respect to the used model and sensors, and relative ease of filter tuning. The authors also tested alternative filters with no practically perceptible estimation improvement. The EKF model in this work consists of a coupled model for the longitudinal and lateral behavior, where the longitudinal behavior is described through a kinematic model and the lateral behavior through a dynamic bicycle model. The longitudinal model is necessary in order to obtain longitudinal velocity and acceleration information which is required for the proper processing of several key variables. Two different approaches for the tire force model are compared. The first approach employs a random walk model for the lateral tire forces (in correspondence with [11]) and this is called the force estimation (FE) approach. In the second approach the lateral tire behavior is included as an adaptive linear tire model, similar to the approach proposed by Baffet et al. [16], [17]. This is called the stiffness estimation (SE) approach. It is demonstrated that combined with the estimated sideslip angles, this approach gives good estimates for the front and rear tire forces. Both approaches are shown to be applicable throughout the nonlinear tire behavior. An observability analysis is performed to evaluate the stability and implementation issues for practical automotive sensors. It is shown that both approaches are locally unobservable during straight driving which can lead to estimator divergence. In order to obtain a stable estimator, even for unobservable configurations, we propose the use of a variable model covariance for the tire stiffness in the SE approach. This approach is proven to provide a stable estimator during straight driving, which is also validated experimentally. However, the same approach cannot be applied to the FE approach. Even though alternative schemes are possible here [26], these are more invasive to the overall estimation approach than the scheme proposed here.

In order to estimate strongly nonlinear tire models, the EKF approach is not adequate as it performs poorly for strong nonlinearities (e.g. the transition to sliding friction). In this

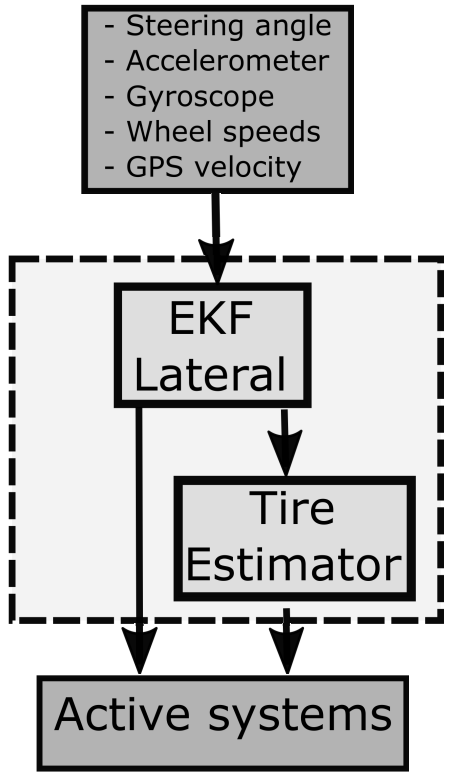

Fig. 1. Structure of the estimator.

work we therefor propose to use a sliding window estimator for the tire model based on the data generated from the EKF. As this estimator needs longer time windows, it offers online instead of real-time performance. From the estimated slip-force behavior, a tire model of choice can be fitted. The parameter estimation for three different tire models is discussed in this work: a bilinear model, a Dugoff model and a 7-parameter magic formula.

The accuracy and robustness of the proposed approach is validated experimentally in mixed driving conditions through experimental tests on a handling track with a commercial vehicle.

The proposed approach provides an easy to implement and extensible setting for vehicle lateral dynamics estimation. A minimal set of measurements is presented which provides good accuracy, but these can be easily extended to more measurements in order to increase the accuracy and/or include additional effects (e.g. banking angle through 2-antenna GPS). Moreover, through the two-stage approach, a range of tire models can be identified, depending on the purpose of the estimator and the available computational power.

This paper gives a brief overview of the two-stage estimator in Sec. II and both stages are detailed further in Sec. III and Sec. IV. The experimental validation results are summarized in Sec. V and the findings of this paper are summarized in Sec. VI.

\section{ESTIMATOR STRUCTURE}

The proposed estimator consists of two main components, as shown in Figure 1. The first part consists of an extended Kalman filter (EKF) which provides information on the (lateral) vehicle behavior based on a dynamic vehicle model. This estimator provides fast response and allows to accurately track the vehicle's state and per-axle lateral tire forces, as discussed in Sec. III. This estimator can provide data in real-time to active vehicle systems due to the efficient EKF formalism. 
The second part consists of a tire parameter estimator which operates on the results of the EKF over a finite time window in order to provide online tire information. This information is processed by fitting a tire model in a leastsquares sense, as discussed in Sec. IV. This estimator receives the estimated tire forces and sideslip angles from the EKF, as well as information on the vertical axle loads based on the acceleration information. Because the information from a longer time-window needs to be processed in an iterative fashion, this estimator is not capable of guaranteeing real-time performance, but can provide online updates. This information is highly valuable for predictive vehicle control. Due to the general framework of this parameter estimation, a wide range of tire models can be fitted (three of which are discussed in this work).

The treatment of this estimation in series allows to exploit the EKF for fast estimation on low cost hardware and mitigates the issues the EKF might have with the highly nonlinear tire models. By using a sliding window estimator for the tire parameters, the full nonlinear tire behavior can be accurately captured and observability issues arising from the joint estimation of a large number of parameters can be effectively mitigated.

\section{JOINT SIDESLIP ANGLE/TIRE-FORCE ESTIMATOR}

This section discusses the joint sideslip angle/tire-force estimator. First, the vehicle models for the estimator are discussed. Next, the discrete-time extended Kalman filter is introduced. Finally an observability analysis is performed for typical commercial vehicle sensors and a variable covariance scheme is proposed to guarantee a stable estimator during straight driving.

\section{A. Vehicle model for the estimator}

The joint sideslip angle/tire-force estimator is based on a dynamic vehicle model. In order to develop an observable system for a common set of automotive sensors, the bicycle model is chosen, as developed by Segel in 1956 [27]. Figure 2 shows this model. The different variables are:

- $\dot{\psi}$ is the yaw rate,

- $v_{x}$ and $v_{y}$ are the longitudinal and lateral center of gravity (COG) vehicle velocities projected onto a vehicle fixed frame and $v$ is the resulting velocity vector,

- $\beta$ is the COG sideslip angle,

- $\alpha_{f}$ and $\alpha_{r}$ are respectively the front and rear wheel sideslip angles,

- $F_{x f}, F_{y f}$ are the longitudinal and lateral front tire force and $F_{x r}, F_{y r}$ are the longitudinal and lateral rear tire force (all described in a tire-attached frame),

- $\delta$ is the steering angle,

- $l_{f}$ and $l_{r}$ are the respective distances from the COG to the front and rear axle.

The choice for the bicycle model is an important one, as it implies that only average tire forces for the front and rear axle can be obtained, rather than separate forces for the left and right side. This is a well considered trade-off, as using a regular four-wheel model would not allow a separation of

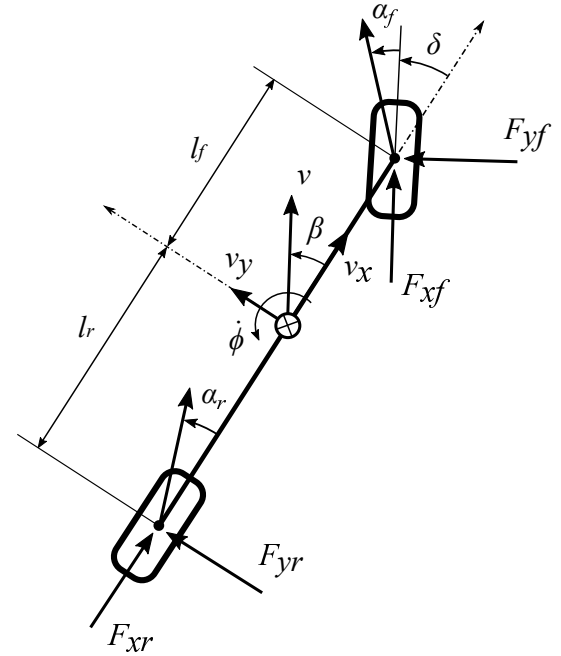

Fig. 2. The bicycle model.

the left and right forces either without prior assumption on this distribution. Furthermore, the assumption is made that the steering angle is small. This decouples the longitudinal and lateral dynamics of the bicycle model and thereby allows to only consider the lateral vehicle dynamics in the estimator. This eliminates the unknown longitudinal tire forces in the model and thus the need for wheel torque measurements in order to obtain an observable system. In this case, the lateral vehicle dynamics can be formulated as:

$$
\left\{\begin{array}{l}
\dot{v}_{y}=\frac{1}{m}\left(F_{y f}+F_{y r}\right)-v_{x} \dot{\psi} \\
\ddot{\psi}=\frac{1}{I_{z z}}\left(l_{f} F_{y f}-l_{r} F_{y r}\right)
\end{array},\right.
$$

where $m$ is the vehicle mass and $I_{z z}$ is the yaw moment of inertia (both of which are assumed known). The longitudinal velocity $v_{x}$ appears in Eq. (1) as an input. In order to properly take its variation into account, the model is augmented with a kinematic update equation for $v_{x}$. This transforms $v_{x}$ into a state variable such that it can be properly estimated from the available measurements. This leads to the following set of coupled model equations:

$$
\left\{\begin{array}{l}
\dot{v}_{y}=\frac{1}{m}\left(F_{y f}+F_{y r}\right)-v_{x} \dot{\psi} \\
\ddot{\psi}=\frac{1}{I_{z z}}\left(l_{f} F_{y f}-l_{r} F_{y r}\right) \\
\dot{v}_{x}=a_{x}+v_{y} \dot{\psi}
\end{array} .\right.
$$

These dynamic equations also require a tire force model. Classic works assumed a known tire model, but this work aims to obtain robustness with respect to variable tire behavior. Two approaches which do not require known tire parameters are discussed and compared in this paper. The first approach consists of a direct estimation of the lateral tire forces by modeling their evolution as a random walk process. This approach will be referred to as the force estimation (FE) approach in the remainder of this work. This approach leads 
to the following continuous-time model for the estimator:

$$
\dot{q}=g_{c}(q, u) \leftrightarrow\left\{\begin{array}{l}
\dot{v}_{y}=\frac{1}{m}\left(F_{y f}+F_{y r}\right)-v_{x} \dot{\psi} \\
\ddot{\psi}=\frac{1}{I_{z z}}\left(l_{f} F_{y f}-l_{r} F_{y r}\right) \\
\dot{v}_{x}=a_{x}+v_{y} \dot{\psi} \\
\dot{F}_{y f}=0 \\
\dot{F}_{y r}=0
\end{array}\right.
$$

with the longitudinal acceleration as input $u=a_{x}$, and state vector $q$ :

$$
q=\left[\begin{array}{c}
v_{y} \\
\dot{\psi} \\
v_{x} \\
F_{y f} \\
F_{y r}
\end{array}\right] .
$$

The second approach describes the lateral tire forces using a simple linear tire model where the resulting axle load due to the lateral tire force $F_{y}$ is given by:

$$
F_{y}=-2 C_{y} \alpha,
$$

with tire cornering stiffness $C_{y}$ and wheel sideslip angle $\alpha$. Under a small angle approximation the front $\left(\alpha_{f}\right)$ and rear $\left(\alpha_{r}\right)$ sideslip angles are given by:

$$
\alpha_{f}=\frac{v_{y}+l_{f} \dot{\psi}}{v_{x}}-\delta, \quad \alpha_{r}=\frac{v_{y}-l_{r} \dot{\psi}}{v_{x}} .
$$

The evolution of the unknown cornering stiffness is in this case modeled as a random walk to account for variable tire behavior (road friction changes, tire nonlinearity, tire wear, etc.). This approach will be referred to as the stiffness estimation (SE) approach in the remainder of this work. Substituting the tire model Eq. (5)-(6) in model Eq. (2) results in the following continuous-time model for the estimator:

$$
\begin{gathered}
\dot{q}=g_{c}(q, u) \leftrightarrow \\
\left\{\begin{array}{l}
\dot{v}_{y}=\frac{-2\left(C_{f}+C_{r}\right)}{m v_{x}} v_{y}-\left(\frac{2\left(C_{f} l_{f}-C_{r} l_{r}\right)}{m v_{x}}+v_{x}\right) \dot{\psi}+\frac{2 C_{f}}{m} \delta \\
\ddot{\psi}=\frac{-2\left(C_{f} l_{f}-C_{r} l_{r}\right)}{I_{z z} v_{x}} v_{y}-\frac{2\left(C_{f} l_{f}^{2}+C_{r} l_{r}^{2}\right)}{I_{z z} v_{x}} \dot{\psi}+\frac{2 C_{f} l_{f}}{I_{z z}} \delta \\
\dot{v}_{x}=a_{x}+v_{y} \dot{\psi} \\
\dot{C}_{f}=0 \\
\dot{C}_{r}=0
\end{array}\right.
\end{gathered}
$$

with input vector $u$ :

$$
u=\left[\begin{array}{c}
\delta \\
a_{x}
\end{array}\right]
$$

and state vector $q$ :

$$
q=\left[\begin{array}{c}
v_{y} \\
\dot{\psi} \\
v_{x} \\
C_{f} \\
C_{r}
\end{array}\right] .
$$

Moreover, it is important to highlight that two sensors are present whose output are used as inputs to the model:

- Longitudinal acceleration measurement $a_{x}$ from an accelerometer.

- Steering angle measurement from a steering mounted encoder, which is combined with a kinematic map of the steering system in order to obtain the left and right wheel angles (respectively $\delta_{l}$ and $\delta_{r}$ ). The wheel angle for the dynamic model is obtained as the average angle of both front wheels:

$$
\delta=\frac{\delta_{l}+\delta_{r}}{2} .
$$

For both approaches, the COG sideslip angle $\beta$ can be computed as:

$$
\beta=\tan ^{-1}\left(\frac{v_{y}}{v_{x}}\right) .
$$

The estimation technique adopted in this work is implemented in discrete-time as this allows for efficient computer implementation. It is thus necessary to time-discretize the model equations Eq. (3) and Eq. (7). Several integration schemes can be exploited to perform this time discretization and a forward Euler integrator is employed here:

$$
\begin{aligned}
q_{k} & =q_{k-1}+g_{c}\left(q_{k-1}, u_{k-1}\right) \Delta t, \\
& =g_{d}\left(q_{k-1}, u_{k-1}\right),
\end{aligned}
$$

where $\Delta t$ is the time step size and subscript $k$ refers to the time sample. In general, the forward Euler scheme has poor stability properties, but this is practically compensated for through the measurement equations correction in the Kalman filter.

\section{B. The augmented extended Kalman filter}

In this work the extended Kalman filter (EKF) is selected for the joint estimation of sideslip angle and lateral tire forces. The EKF is a straightforward extension of the linear Kalman filter by considering a linearization of the nonlinear system around the current configuration [25]. The system equations of motion Eq. (12) are complemented by the (nonlinear) measurement equations:

$$
w_{k}=h\left(q_{k}, u_{k}\right)+r_{w},
$$

in which $w_{k}$ contains the sensor measurements as obtained from the measurement equations $h$ corrupted by measurement noise $r_{w}$. The noise is assumed to be zero-mean uncorrelated Gaussian noise with covariance matrix $R$. Like the linear Kalman filter, the EKF algorithm is recursive and operates in two steps:

- The time update step predicts the current system state $\hat{q}_{k}^{-}$ and propagates the state error covariance $P_{k}^{-}$:

$$
\begin{gathered}
\hat{q}_{k}=g_{d}\left(\hat{q}_{k-1}^{+}, u_{k-1}\right), \\
P_{k}^{-}=G_{d, k-1} P_{k-1}^{+} G_{d, k-1}^{T}+Q_{k-1} .
\end{gathered}
$$

- Once the measurement $w_{k}$ is available, the Kalman gain $K_{k}$ is calculated which is then used to update the system state estimate $\hat{q}_{k}^{+}$by incorporating the information available in the measurement. The definition of the Kalman gain $K_{k}$ originates from the minimization of the trace of the state error covariance matrix $P_{k}^{+}$. Lastly, the updated state error covariance $P_{k}^{+}$is computed:

$$
\begin{gathered}
K_{k}=P_{k}^{-} H_{k}^{T}\left(H_{k} P_{k}^{-} H_{k}^{T}+R\right)^{-1}, \\
\hat{q}_{k}^{+}=\hat{q}_{k}^{-}+K_{k}\left(w_{k}-h\left(\hat{q}_{k}^{-}, u_{k}\right)\right), \\
P_{k}^{+}=\left(I-K_{k} H_{k}\right) P_{k}^{-} .
\end{gathered}
$$


In both the time- and measurement update step, the propagation of the state error covariance is performed by linearizing, respectively, the (discrete) model- and measurement equations around the current configuration:

$$
\begin{aligned}
G_{d, k-1} & =\left.\frac{\partial g_{d}}{\partial q}\right|_{q_{k-1}, u_{k-1}}, \\
H_{k} & =\left.\frac{\partial h}{\partial q}\right|_{q_{k}, u_{k}} .
\end{aligned}
$$

This estimator has the potential to run in real-time as the overall method has a deterministic computational load. All the model evaluations have a fixed computational load and the overall model is small enough such that no iterative methods are required to evaluate the matrix inverses for the Kalman filter. The authors have also implemented the estimation algorithm on a real-time dSPACE system to demonstrate the real-time capabilities [28].

\section{Observability analysis and stabilization}

For sequential estimation problems like the Kalman filter, the observability is a key property as it determines whether a state can be estimated and whether the estimator remains stable. The observability always depends on the combination of model equations and measurement equations. In this work four different types of measurements are considered:

- Yaw rate measurement from a (MEMS) gyroscope:

$$
\dot{\psi}=\dot{\psi} .
$$

- Lateral acceleration measurement from a (MEMS) accelerometer. For the FE model, the measurement equation becomes:

$$
a_{y}=\frac{1}{m}\left(F_{y f}+F_{y r}\right),
$$

and for the SE model the measurement equation becomes:

$$
a_{y}=\frac{-2\left(C_{f}+C_{r}\right)}{m v_{x}} v_{y}-\frac{2\left(l_{f} C_{f}-l_{r} C_{r}\right)}{m v_{x}} \dot{\psi}+\frac{2 C_{f}}{m} \delta .
$$

- Four wheel speed measurements from wheel mounted encoders:

$$
\begin{aligned}
v_{r l} & =v_{x}-\frac{t_{r}}{2} \dot{\psi} \\
v_{r r} & =v_{x}+\frac{t_{r}}{2} \dot{\psi} \\
v_{f l} & =\left(v_{x}-\frac{t_{f}}{2} \dot{\psi}\right) \cos \left(\delta_{l}\right)+\left(v_{y}+l_{f} \dot{\psi}\right) \sin \left(\delta_{l}\right), \\
v_{f r} & =\left(v_{x}+\frac{t_{f}}{2} \dot{\psi}\right) \cos \left(\delta_{r}\right)+\left(v_{y}+l_{f} \dot{\psi}\right) \sin \left(\delta_{r}\right),
\end{aligned}
$$

with $t_{r}$ and $t_{f}$ respectively the rear and front track width. It is important to highlight that these equations only hold for limited longitudinal tire slip. These measurements are already available on all vehicles with ESC systems.

- GPS velocity measurement (available from most commercial sensors):

$$
v_{g p s}=\sqrt{v_{x}^{2}+v_{y}^{2}} .
$$

This leads to the full measurement set:

$$
w=h(q, u)=\left[\begin{array}{c}
\dot{\psi}(q, u) \\
a_{y}(q, u) \\
v_{r l}(q, u) \\
v_{r r}(q, u) \\
v_{f l}(q, u) \\
v_{f r}(q, u) \\
v_{g p s}(q, u)
\end{array}\right] .
$$

For nonlinear estimation problems with an extended Kalman filter approach both global and local observability need to be considered. Global observability can be analyzed through the evaluation of the Lie derivatives [29] and it can be shown for the above equations that the combination of these models and measurement leads to a globally observable system. However, for the extended Kalman filter to be stable a sufficient condition is the local observability of the system [30] and global observability is not sufficient. In this work, the PopovBelevitch-Hautus (PBH) test of observability is applied on the linearized continuous-time system [31] for the evaluation of the local observability. This criterion states that the linearized system is observable if and only if the matrix:

$$
\mathcal{O}_{P B H}(s)=\left[\begin{array}{c}
G_{c}-s I \\
H
\end{array}\right],
$$

is of full rank for all $s \in \mathbb{C}$, where $G_{c}$ is the Jacobian of the continuous-time model Eq. (3) or (7), and $H$ is the Jacobian of the measurement equations Eq. (29) for a given state. As the dynamic model equations are of full rank for all $s$ except for the eigenvalues of the system it is sufficient to check the rank of $\mathcal{O}_{P B H}(s)$ for these eigenvalues $\lambda$ of the system matrix $G_{c}$. The eigenvalues of $G_{c}$ are the eigenvalues of the original linearized system appended with a double zero eigenvalue due to the random walk model for the unknown lateral tire forces or cornering stiffnesses. For the above described system following remarks can be made based on the PBH-criterion:

- For the FE approach: if the car is driving in a straight line $\left(\delta=\dot{\psi}=a_{y}=v_{y}=0\right)$, the lateral velocity $v_{y}$ is unobservable irrespective of which of the above measurements is used. This can lead to erratic behavior of the lateral velocity when this should be zero.

- For the SE approach: if the car is driving in a straight line $\left(\delta=\dot{\psi}=a_{y}=v_{y}=0\right)$, the cornering stiffnesses $C_{y i}$ are unobservable irrespective of which of the above measurements are used. This can be expected as there is no information about the lateral dynamics in this case, allowing the cornering stiffnesses to have any value while producing the same measurement outputs. This translates into a random drift of the cornering stiffnesses during straight driving.

- For the FE and SE approach: if the car is cornering, at least the yaw rate and acceleration measurements are required in order to obtain an observable system. However, the fusion with the wheel speed measurements and/or GPS velocity measurement provides valuable additional information which leads to better estimation accuracy by lowering the estimation covariance and because they prevent drift due to accelerometer bias. 
The above remarks indicate that stability issues can occur during (prolonged) straight driving for both the FE and SE approach. However, this local observability is a sufficient but not a necessary condition for a stable Kalman filter. As a necessary condition the (discrete) Riccati equation ${ }^{1}$ needs to be able to converge to a steady solution [32]:

$$
P=G_{d} P G_{d}^{T}-\left(G_{d} P H^{T}\right)\left(R+H P H^{T}\right)^{-1}\left(H P G_{d}^{T}\right)+Q .
$$

The model covariance $Q$ for a vehicle is very difficult to determine in practice as this is typically strongly statedependent. It will also contain many (biased) model errors due to the strong model simplification of the bicycle model. The model covariance $Q$ is therefor practically tuned in order to yield the best results for a set of reference tests. From this point of view the model covariance $Q$ can also be exploited as a final tuning parameter to stabilize the estimator, even though this undermines the physical interpretation of the model covariance $Q$. This is the approach which we will use in this work to create an unconditionally stable estimator for lateral vehicle dynamics. Here it is important that these adjustments do not limit the general operation of the estimator. In order to show how the model covariance can be exploited, we consider a partition of the states of the FE model for the unobservable state of straight driving:

$$
q_{F E}=\left[\begin{array}{l}
q_{u} \\
q_{o}
\end{array}\right],
$$

with $q_{u}=v_{y}$ the unobservable state and $q_{o}$ the other observable states. The state transition matrix for the FE model $G_{d, F E}$ can then be written as:

$$
G_{d, F E}=\left[\begin{array}{ll}
I & G_{d, u o} \\
0 & G_{d, o o}
\end{array}\right],
$$

and the measurement Jacobian becomes:

$$
H_{F E}=\left[\begin{array}{ll}
0 & H_{o}
\end{array}\right] \text {. }
$$

Similarly, the states for the SE approach can be written as:

$$
q_{S E}=\left[\begin{array}{l}
q_{o} \\
q_{u}
\end{array}\right]
$$

with $q_{u}=\left[C_{f}, C_{r}\right]^{T}$ the unobservable states and $q_{o}$ the other observable states. The state transition matrix for the SE model $G_{d, S E}$ becomes:

$$
G_{d, S E}=\left[\begin{array}{cc}
G_{d, o o} & 0 \\
0 & I
\end{array}\right],
$$

and the measurement Jacobian becomes:

$$
H_{S E}=\left[\begin{array}{ll}
H_{O} & 0
\end{array}\right] \text {. }
$$

First we evaluate the Riccati equation for the SE approach. Following Eq. (35), the estimation covariance can be written as:

$$
P=\left[\begin{array}{ll}
P_{o o} & P_{o u} \\
P_{u o} & P_{u u}
\end{array}\right],
$$

${ }^{1}$ We omit the $k$ subscript in this derivation for simplicity. and the Riccati equation becomes:

$$
\begin{aligned}
& P_{o o}=G_{d, o o} P_{o o} G_{d, o o}^{T}-G_{d, o o} P_{o o} H_{o}^{T}\left(R^{*}\right)^{-1} H_{o} P_{o o} G_{d, o o}^{T}+Q_{o o}, \\
& P_{o u}=G_{d, o o}^{T} P_{o u}-G_{d, o o} P_{o o} H_{o}^{T}\left(R^{*}\right)^{-1} H_{o} P_{o u}, \\
& P_{u o}=P_{u o} G_{d, o o}-P_{u o} H_{o}^{T}\left(R^{*}\right)^{-1} H_{o} P_{o o} G_{d, o o}^{T}, \\
& P_{u u}=P_{u u}-P_{o u}^{T} H_{o}^{T}\left(R^{*}\right)^{-1} H_{o} P_{o o} P_{u o}+Q_{u u},
\end{aligned}
$$

with

$$
R^{*}=R+H P H^{T}
$$

This Riccati equation always has a solution for $P_{o o}$ as this part of the equation is a regular observable system. The other parts of the equation have a neutrally stable solution $P_{o u}=$ $P_{u o}=0$ for any constant $P_{u u}$ if $Q_{u u}=0$. This show that $Q$ can indeed be used to obtain a stable estimator for the locally unobservable system. In order to accomplish this, we define a variable model covariance such that $Q_{u u}=0$ for straight driving, but not for the other states. By employing this approach we obtain a smooth transition between the different regimes. In this case we express $Q$ as a function of the steering angle $\delta$. Several choices for this variation of the covariance are possible. In this work we propose to use a logarithmic function of the steering angle:

$$
Q_{k-1}^{S E}=\left[\begin{array}{ccccc}
Q_{v_{y}} & 0 & 0 & 0 & 0 \\
0 & Q_{\psi} & 0 & 0 & 0 \\
0 & 0 & Q_{v_{x}} & 0 & 0 \\
0 & 0 & 0 & Q_{C_{f}}^{0} \log _{10}\left(9 \frac{\left\|\delta_{k-1}\right\|}{\delta_{\text {max }}}+1\right) & 0 \\
0 & 0 & 0 & 0 & Q_{C_{r}}^{0} \log _{10}\left(9 \frac{\left\|\delta_{k-1}\right\|}{\delta_{\max }}+1\right)
\end{array}\right]
$$

This specific dependance on the steering angle $\delta$ always leads to a positive covariance matrix where the covariance for the unobservable states goes to zero as the steering angle approaches zero. For all states which are always locally observable the covariance is fixed. For the locally nonobservable states a maximum covariance value is defined. This dependance also allows for higher uncertainty under large sideslip angles which enables the estimator to better track the the strongly nonlinear tire behavior in this regime. The use of the steering angle allows a straightforward normalization, as the lock-to-lock steering angle $\delta_{\max }$ is known in advance. This allows for a straightforward tuning of the covariances $Q_{C_{f}}^{0}$ and $Q_{C_{r}}^{0}$. This stabilizability is an interesting observation because even though both approaches are seemingly equivalent, the SE approach allows for a straightforward stabilization in unobservable conditions. Other functions (like a quadratic dependance) are also possible for the variable covariance, as long as the function goes to zero for the unobservable state. However, for the cases presented in Sec. V we obtained the best results with the presented logarithmic function. It is interesting to notice that this adaptive covariance matrix has no impact on the real-time capabilities of the proposed estimator, as the cost of evaluating this covariance matrix is negligble.

However, in the case of the FE approach no simple relation exist to obtain a steady Riccati equation due to the more coupled nature of $F_{F E}$ in Eq. (33). For this reason the FE approach cannot be stabilized simply by varying $Q$. This is a drawback of the FE approach with respect to the SE approach, which is also clearly visible in the results obtained in Sec. V. 
It is important to distinguish the proposed approach from adaptive Kalman filtering methods [33]. In the latter, the covariance is determined online such that no solution is offered with respect to stabilization. In this work however, we inherently exploit the system knowledge where we know a-priori that for the proposed model, the system should not exhibit lateral dynamics when no steering input is present. Sec. V shows that this approach provides accurate estimation results with a stable estimator (in contrast to the estimator with constant covariance).

\section{NONLINEAR LEAST-SQUARES TIRE PARAMETER} ESTIMATOR

In the second part of the estimator, the tire parameters are estimated. For the tire parameter estimation, the estimated sideslip angle (together with the steering angles) is combined with the estimated tire forces, in order to fit a tire model. For this estimation we will not exploit a sequential approach. This can lead to incorrect variables related to the friction limit of the tire, as these only have a strong impact when relatively large excitation is present. In this part of the estimator we will use an optimization based fitting over a finite time window. Here it is important to choose the window sufficiently large in order to ensure that a sufficient amount of the nonlinear tire behavior is captured. The tire parameter estimation is performed online rather than in real-time. As the EKF has a fixed computational load, it can be guaranteed to run concurrently with realtime. However the iterative method used for fitting the tire model does not have predetermined computational load (if an accuracy based stopping criterion is used). On average this approach is sufficiently cheap to run in real-time, but overshoots are possible, leading to online behavior.

In this work we apply a general nonlinear least-squares fitting method for the tire parameters. This leaves us with a very flexible framework to estimate different tire models. In section $\mathrm{V}$, it is shown that this approach leads to fast convergence in practice. Three lateral tire models are considered in this work:

- Bilinear tire model: the bilinear tire model is the most basic model which allows to account for saturation of the tire. This is a simple physics based model which assumes the tire behaves linearly until the friction limit is reached. The tire force $\left(F_{t}\right)$ equations for this model are given by:

$$
F_{t}(\alpha, N, p)=\left\{\begin{array}{ll}
C \alpha, & \alpha \leq(N \mu / C) \\
N \mu, & \alpha>(N \mu / C)
\end{array} .\right.
$$

In this equation $\alpha$ is the sideslip angle of the tire and $N$ is the normal tire load. Two parameters need to be determined, being the linear tire stiffness $C$ and the friction coefficient $\mu$, such that:

$$
p=\left[\begin{array}{l}
C \\
\mu
\end{array}\right] .
$$

The Jacobian $J_{p}$ of the tire force with respect to these parameters is straightforward to compute and can readily be vectorized for different sideslip-angles:

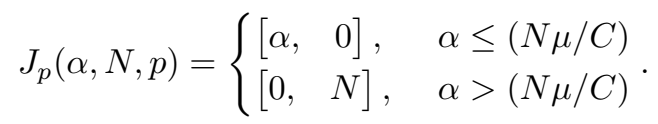

These derivatives can give issues if a state is exactly at the transition, but this is statistically unlikely, such that it is not an issue in practice. This easy to evaluate Jacobian allows a particularly efficient fitting.

- Dugoff tire model: this model is another basic physics inspired tire model with only two parameters, but which provides a smooth transition from the linear tire operation to the saturation behavior [34], [35]. The governing equations for the lateral tire model (assuming zero longitudinal slip) are:

$$
\begin{gathered}
\lambda= \begin{cases}\frac{N \mu}{2 C \tan (\alpha)}, & \alpha \neq 0 \\
1, & \alpha=0\end{cases} \\
F_{t}(\alpha, N, p)= \begin{cases}C \tan (\alpha)\left(2 \lambda-\lambda^{2}\right), & \lambda<1 \\
C \tan (\alpha), & \lambda \leq 1\end{cases}
\end{gathered}
$$

In this formula, $\lambda$ determines whether saturation occurs, but the course of the saturation behavior cannot be adjusted through the two parameters, being the linear lateral tire stiffness $C$ and the tire-road friction $\mu$. The parameters $p$ to fit are then:

$$
p=\left[\begin{array}{l}
C \\
\mu
\end{array}\right] .
$$

It is interesting to notice that even though this is the same parameter set as the bilinear model, the fitting process is much more nonlinear. This will also be reflected in the computational load, as discussed in Sec. V. Again, the Jacobian $J_{p}$ can be readily evaluated:

$$
J_{p}(\alpha, N, p)=\left\{\begin{array}{ll}
{\left[\begin{array}{rl}
\tan (\alpha)\left(\left(2 \lambda-\lambda^{2}\right)+C(2-2 \lambda) \frac{\partial \lambda}{\partial C}\right), \\
C \tan (\alpha)\left(2 \frac{\partial \lambda}{\partial \mu}-2 \lambda \frac{\partial \lambda}{\partial \mu}\right)
\end{array}\right],} & \lambda<1 \\
{[\tan (\alpha),} & 0],
\end{array},\right.
$$

with

$$
\begin{aligned}
& \frac{\partial \lambda}{\partial C}=\left\{\begin{array}{ll}
\frac{-N \mu \tan (\alpha)}{8(C \tan (\alpha))^{2}}, & \alpha \neq 0 \\
0, & \alpha=0
\end{array},\right. \\
& \frac{\partial \lambda}{\partial \mu}= \begin{cases}\frac{N}{2 C \tan (\alpha)}, & \alpha \neq 0 \\
0, & \alpha=0\end{cases}
\end{aligned}
$$

Again these derivatives can lead to issues in the case where an estimated state is exactly at a transition point of the function, but in practice these cases are negligible and have limited impact when many measurement points are available.

- Magic formula: the magic tire formulas proposed by Pacejka [36] are popular data based tire models. These models allow to fit a wide range of effects by introducing more parameters. Unfortunately these parameters lack the direct physical interpretation of the other two models but offer the possibility to model the transition from static to sliding friction, which is of paramount importance e.g. in ABS control. In this work, the most basic magic formula employing four parameters is used:

$$
F_{t}(\alpha, N, p)=(N D) \sin (C \operatorname{atan}(B(1-E) \alpha+\operatorname{atan}(B \alpha))),
$$


with four unknown parameters $p$ :

$$
p=\left[\begin{array}{l}
B \\
C \\
D \\
E
\end{array}\right]
$$

Also here the Jacobian $J_{p}$ (given in Eq. (56), see next page) can be readily obtained. The advantage over the previous models is the fact that this function is differentiable as it is fully continuous. This will also be reflected in the speed of convergence we see in Sec. V.

Many other tire models can be used in this context, but these three basic models provide a good trade-off with respect to accuracy and number of parameters, which will influence the computational load of the parameter estimation.

These three tire models require a normal tire load. As the used bicycle model does not distinguish between the effect of the right and left tire during cornering, the lateral load transfer is not taken into account. This prevents a considerable source of error for the first stage estimator as the (dynamic) roll stiffness characteristics can be difficult to identify accurately. The load transfer is therefore only based on the fore-aft transfer with the measured accelerations:

$$
\begin{aligned}
N_{f} & =m g \frac{l_{r}}{l_{r}+l_{f}}-a_{x} m h_{C O G}, \\
N_{r} & =m g \frac{l_{f}}{l_{r}+l_{f}}+a_{x} m h_{C O G} .
\end{aligned}
$$

In these equations $g$ is the gravitational acceleration and $h_{C O G}$ is the height of the COG with respect to the road. This height is assumed approximately constant, as detailed suspension models for evaluating the change in height are typically not available and would greatly complicate the approach.

In order to solve the fitting problem, a mean least squares fitting problem is solved. It is a conscious choice in this work to not perform a weighted least squares problem exploiting the variance from the EKF, as these values are known to not be reliable in general [37]. Moreover, in this work they are extended by other uncertainties like the normal tire load. The minimization problem to solve is:

$$
\min _{p \in \mathbb{R}^{m}} \frac{1}{2}\left\|F\left(\alpha_{e s t}(t), N(t), p\right)-F_{y}^{e s t}(t)\right\|_{2}^{2} \quad t \in\left[t_{1}, t_{2}\right],
$$

where the parameter vector $p$ depends on the tire model used, as described above. This optimization is performed for the data over a time-frame from $t_{1}$ to $t_{2}$. This window is to be used as a tuning parameter where several aspects have to be taken into account:

- A very long window will probably capture more of the tire behavior, thus ensuring a reliable fitting of the saturation parameters.

- As the tire behavior is varying in time due to varying road conditions and wear, the window should not be too long to ensure that a constant tire model does not need to account for the time varying (rather than nonlinear) tire behavior.

- The longer the window, the higher the computational load, but this is only a linear dependance.
- The time window should be long enough in order to include a sufficient amount of cornering phenomena as straight driving does not yield relevant information for the tire fitting (and can even be omitted from the fitting data in order to reduce the computational load).

In general, it is difficult to devise exact rules for this window. In particular applications however, sensible choices are possible. For the case of track driving, the window can for example be adjusted to the length of single lap.

Many methods exist to solve nonlinear least-squares problems in general [38], and in this work a trust-region GaussNewton approach is adopted. This algorithm is summarized for this application in Algo. 1. As the number of parameters

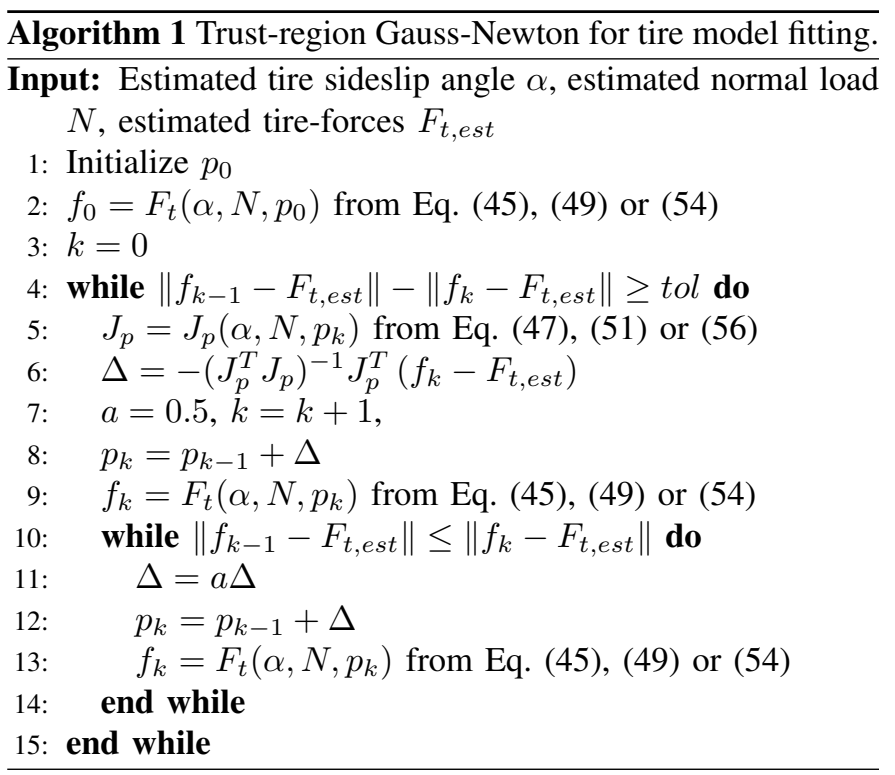

for most tire-models are relatively limited (less than ten), the inversion of the squared Jacobian does not lead to large computational loads. The optimization is terminated when negligible improvement is obtained for the fit. For nonlinear optimization, the initial guess for the solution is of paramount importance. As the optimization problem in this work is solved in a sliding window setting, the results from the previous window can be used to initialize the algorithm, ensuring a proper initial guess and fast convergence. When no other reference is available, both the bilinear and Dugoff tire model assume the same initial parameters $p_{0}$ :

$$
\begin{aligned}
& \mu_{0}=\max \left(F_{t, e s t}\right) / N \\
& C_{0}=\max \left(F_{t, e s t}\right) / \alpha .
\end{aligned}
$$

For the magic formula, a generic set of initial parameters is used:

$$
p_{0}=\left[\begin{array}{c}
10 \\
1.9 \\
1 \\
0.97
\end{array}\right]
$$

For the general validation cases presented hereafter, these initial conditions lead to fast convergence. 


$$
J_{p}(\alpha, N, p)=\left[\begin{array}{c}
\left.(N D) \cos (C \operatorname{atan}(B(1-E) \alpha+E \operatorname{atan}(B \alpha))) \frac{C}{1+(B(1-E) \alpha+E \operatorname{atan}(B \alpha))^{2}}\right)\left((1-E) \alpha+\frac{E}{1+(B \alpha)^{2}} \alpha\right) \\
(N D) \cos (C \operatorname{atan}(B(1-E) \alpha+E \operatorname{atan}(B \alpha))) \operatorname{atan}(B(1-E) \alpha+E \operatorname{atan}(B \alpha)) \\
N \sin (C \operatorname{atan}(B(1-E) \alpha+E \operatorname{atan}(B \alpha))) \\
(N D) \cos \left(C \operatorname{atan}(B(1-E) \alpha+E \operatorname{atan}(B \alpha)) \frac{C}{1+(B(1-E) \alpha+E \operatorname{atan}(B \alpha))^{2}}(-B \alpha+\operatorname{atan}(B \alpha))\right.
\end{array}\right]^{T}
$$

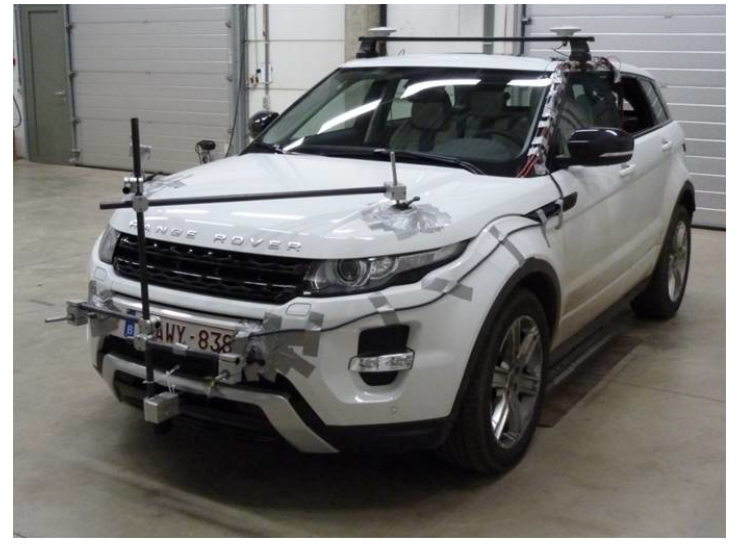

Fig. 3. Flanders Make test vehicle: Range Rover Evoque.

\section{VALIDATION}

The above proposed estimators are validated experimentally on a test vehicle. The test vehicle is a Range Rover Evoque provided by Flanders Make, see Figure 3. For the tests, the vehicle is equipped with several sensors: a lowcost Global Navigation Satellite System (GNSS) receiver, an inertial measurement unit (IMU) and a Corrsys Datron optical sensor. The IMU is a 6 degree-of-freedom sensor chip with integrated accelerometer and gyroscope, sampled at $100 \mathrm{~Hz}$ (SBG Systems IG-500A [39]). In this IMU an internal EKF is applied to remove sensor bias and the IMU is located close to the actual COG, such that centripetal bias is negligible. The GNSS receiver is a low-cost device which provides a velocity measurement at a rate of $1 \mathrm{~Hz}$. The Corrsys Datron CORREVIT S-350 optical correlation sensor measures the vehicle longitudinal and lateral velocity with respect to the ground. This sensor is a high-cost device and serves to validate the estimation results. Furthermore, also the integrated Electronic Stability Control (ESC) system sensors are logged: the steering wheel angle and the wheel speeds. The vehicle parameters necessary for evaluating the models and measurement equations are presented in table I.

TABLE I

VeHICLE MOdel PARAMETERS OF THE RANGe ROVER EVOQUE

\begin{tabular}{lllr}
\hline$m$ & Vehicle mass & 2068 & $\mathrm{~kg}$ \\
$I_{z z}$ & Yaw moment of inertia & 3231 & $\mathrm{kgm}^{2}$ \\
$l_{f}$ & Distance between COG and front axle & 1.077 & $\mathrm{~m}$ \\
$l_{r}$ & Distance between COG and rear axle & 1.583 & $\mathrm{~m}$ \\
$t_{f}$ & Track width of front axle & 1.625 & $\mathrm{~m}$ \\
$t_{r}$ & Track width of rear axle & 1.625 & $\mathrm{~m}$ \\
$h_{C O G}$ & Height of COG & 0.65 & $\mathrm{~m}$ \\
\hline
\end{tabular}

The kinematic relationship between the steering wheel angle and the steering angle of the front left and front right wheel

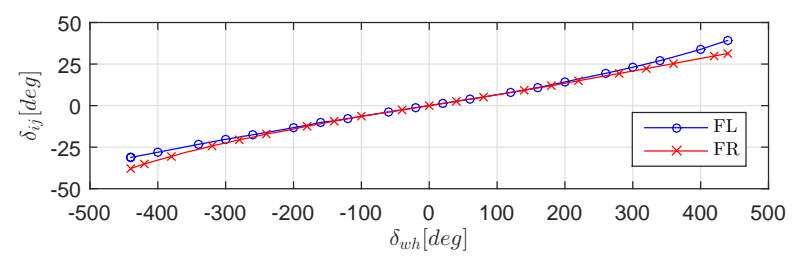

Fig. 4. The relationship steering wheel angle - steering angle.

is shown in Figure 4.

\section{A. Estimator tuning}

For the covariance tuning of the Kalman filter, the lateral bicycle model is assumed exact $\left(Q_{v_{y}}=Q_{\dot{\psi}}=0\right)$ and all uncertainty is assumed on the random walk models for the lateral tire forces and cornering stiffnesses. This is a reasonable assumption as the unknown lateral tire forces or cornering stiffnesses will dominate the uncertainty in the model. The estimated covariance therefor quickly converges to a matrix dominated by the tire force/cornering stiffness model covariance. The relatively small model covariance for the other states has a negligible impact on this estimated covariance and can therefor be omitted. Some uncertainty is also assumed on the longitudinal velocity update to account for accelerometer bias. The resulting full estimated covariance will allow correction of all states based on the measurements. The model covariances for the FE approach are chosen as:

$$
Q_{v_{x}}=1 \cdot 10^{-4}(\mathrm{~m} / \mathrm{s})^{2}, \quad Q_{F_{y f}}=Q_{F_{y r}}=4 \cdot 10^{4} \mathrm{~N}^{2} .
$$

For the SE approach with constant covariance, this is chosen as:

$Q_{v_{x}}=1 \cdot 10^{-4}(\mathrm{~m} / \mathrm{s})^{2}, \quad Q_{C_{f}}=Q_{C_{r}}=25 \cdot 10^{4}(\mathrm{~N} / \mathrm{rad})^{2}$, and for the stabilized SE approach the covariance parameters are:

$Q_{v_{x}}=1 \cdot 10^{-4}(\mathrm{~m} / \mathrm{s})^{2}, \quad Q_{C_{f}}^{0}=Q_{C_{r}}^{0}=125 \cdot 10^{4}(\mathrm{~N} / \mathrm{rad})^{2}$, with $\delta_{\max }=0.25 \mathrm{rad}$.

The measurement covariance matrix $R$ contains the sensor noise variances. These values were obtained from a range of reference measurements:

$$
\begin{gathered}
R_{\dot{\psi}}=1.8 \cdot 10^{-5}(\mathrm{rad} / \mathrm{s})^{2}, \quad R_{a_{y}}=3.1 \cdot 10^{-3}\left(\mathrm{~m} / \mathrm{s}^{2}\right)^{2} \\
R_{v_{i j}}=4.7 \cdot 10^{-4}(\mathrm{~m} / \mathrm{s})^{2}, \quad R_{v_{G P S}}=5(\mathrm{~m} / \mathrm{s})^{2}
\end{gathered}
$$

Finally, the state vector $\hat{q}$ and the state error covariance matrix $P$ need to be initialized. For the state vector, the lateral velocity and the yaw rate are initialized at zero, and the longitudinal velocity is initialized at the average of the four wheel speeds. The lateral tire forces are initialized at $0 N$, 


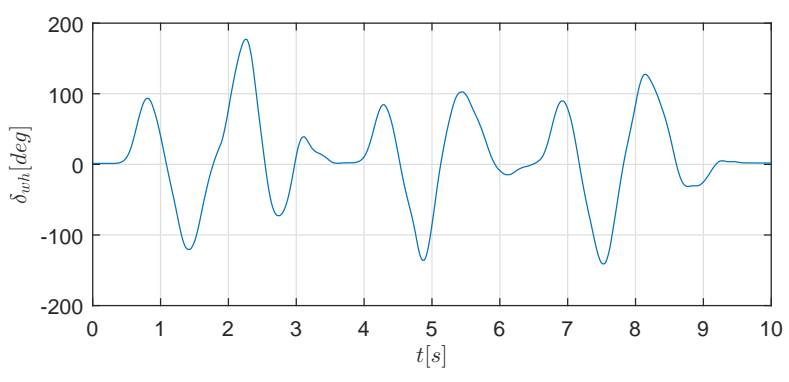

Fig. 5. Steering input for the double lane change.

and the cornering stiffnesses at $60000 \mathrm{~N} / \mathrm{rad}$ for the front and rear. The state error covariance matrix is initialized as the unity matrix.

\section{B. Results and discussion}

The proposed two-stage estimator is validated experimentally for two different test scenarios: ${ }^{2}$

- a double lane change,

- a dedicated handling track. ${ }^{3}$

The performance of the FE and SE (with variable model covariance) approaches are compared for these two scenarios in this section. Both the state-estimation accuracy and the reliability of the tire parameter estimation are discussed. In both cases the SE approach shows considerably more reliable results than the FE approach.

1) Double lane change: A double lane change represents an obstacle avoidance maneuver. This test is chosen because it involves high sideslip angles and a persistent steering input. For the experimental test, three consecutive double lane changes were performed. Figure 5 shows the steering input for this maneuver. Figure 6 summarizes the available measurements for the estimator: the longitudinal and lateral acceleration, and the four wheel speeds. No GPS data was collected as the limited range of this maneuver together with the high covariance for the GPS measurement would lead to negligible benefits in terms of accuracy. The yaw rate measurement is shown in Figure 7 together with its estimate for both estimation approaches. Figure 8 shows the estimated longitudinal and lateral velocity, and compares them to the Corrsys Datron measurement (reference). From these velocities the sideslip angle can be obtained which is shown in Figure 9. From these figures it is clear the SE approach provides considerably better estimates for this maneuver than the FE approach. Due to the persistent excitation for this maneuver, there are no observability issues and the estimation covariances remain stable. The estimated lateral tire forces as a function of time are depicted in Figure 10. This figure shows some important differences between both estimators, but it is difficult to determine which of these results is better than the other from this comparison.

\footnotetext{
${ }^{2}$ These test were not performed on the same day.

${ }^{3}$ The handling track at the Ford Lommel Proving Ground (LPG) in Lommel, Belgium.
}
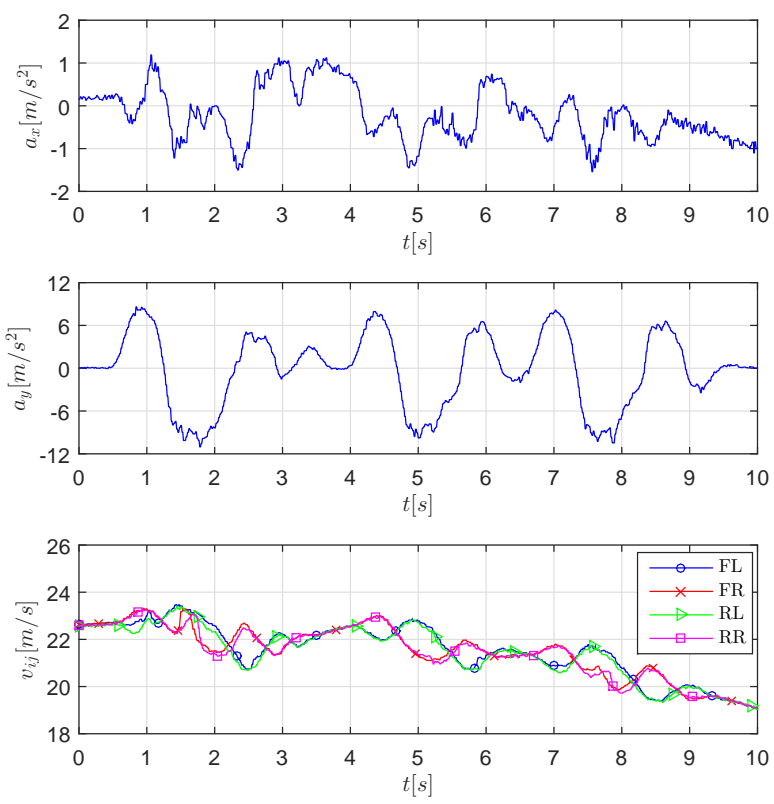

Fig. 6. Overview of the measurements for the double lane change. Top to bottom: longitudinal acceleration, lateral acceleration, and wheel speeds.

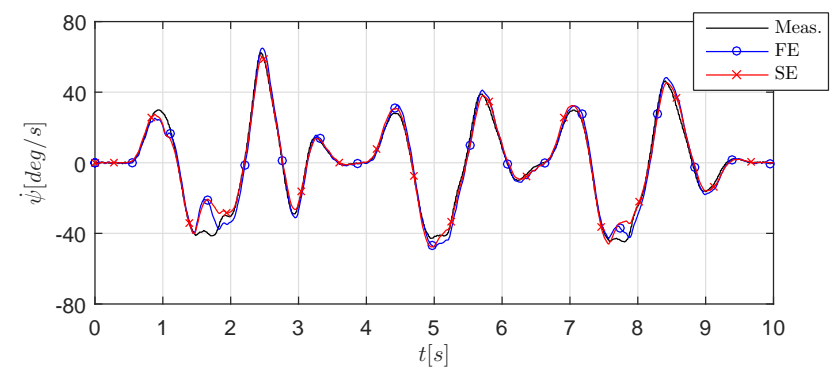

Fig. 7. Yaw rate for the double lane change: measurement (IMU) and estimate for both estimation approaches.
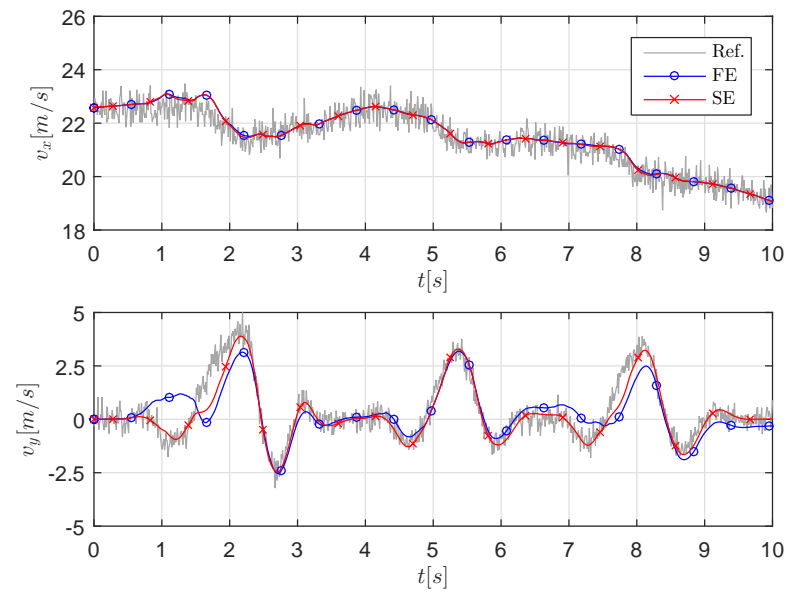

Fig. 8. Longitudinal and lateral velocity for the double lane change: Corrsys Datron measurement (reference) and estimate for both estimation approaches. 


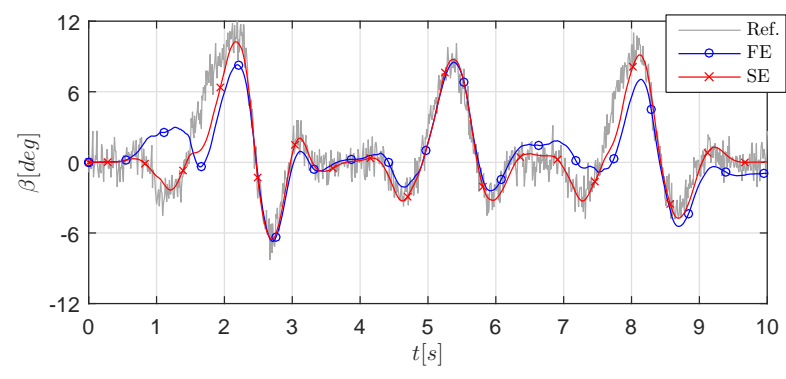

Fig. 9. Sideslip angle for the double lane change: Corrsys Datron measurement (reference) and estimate for both estimation approaches.
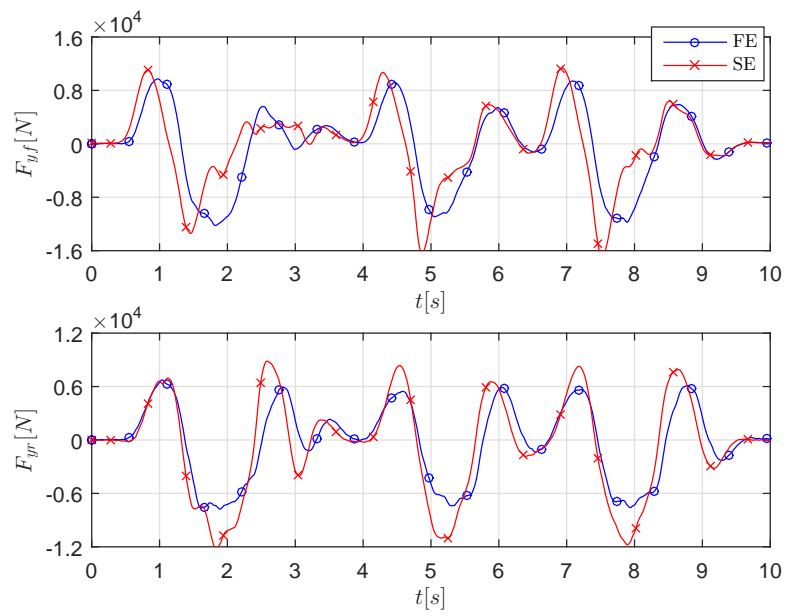

Fig. 10. The estimated per-axle lateral tire forces for the double lane change for both estimation approaches.

In order to get a better view on the accuracy of the estimated forces we will consider the tire model estimation. The estimated wheel sideslip angles and the estimated forces from Figure 10 are combined in order to create a slipforce characteristic from which the tire parameters can be estimated. The results are summarized in Figures 11 and 12 for respectively the front and rear tires. The plots at the top show the slip-force characteristic for the FE and SE approach. For the FE approach, it is immediately apparent that the obtained characteristic diverges quite strongly from the ideal tire characteristics, which makes the fitting of a valid tire model relatively difficult and indicates an unreliable estimate. The SE approach on the other hand appears to deliver much more reliable results and the three tire models from Sec. IV can provide an accurate fit to the estimated forces, as shown in the bottom figures of Fig. 11 and Fig. 12. The number of iterations required to obtain the tire model parameters are summarized in table II. This data was fitted on 1050 data points. Due to the low number of iterations, this fitting can easily be performed online. It is also interesting to notice that the Pacejka model has relative difficulties to fit a very close to linear model (in the case of the front tire) due to its inherent strongly nonlinear nature.

2) Ford LPG handling track: The handling track at Ford LPG is a $4.3 \mathrm{~km}$ long track with sharp turns in different directions and several straight driving sections. This track is
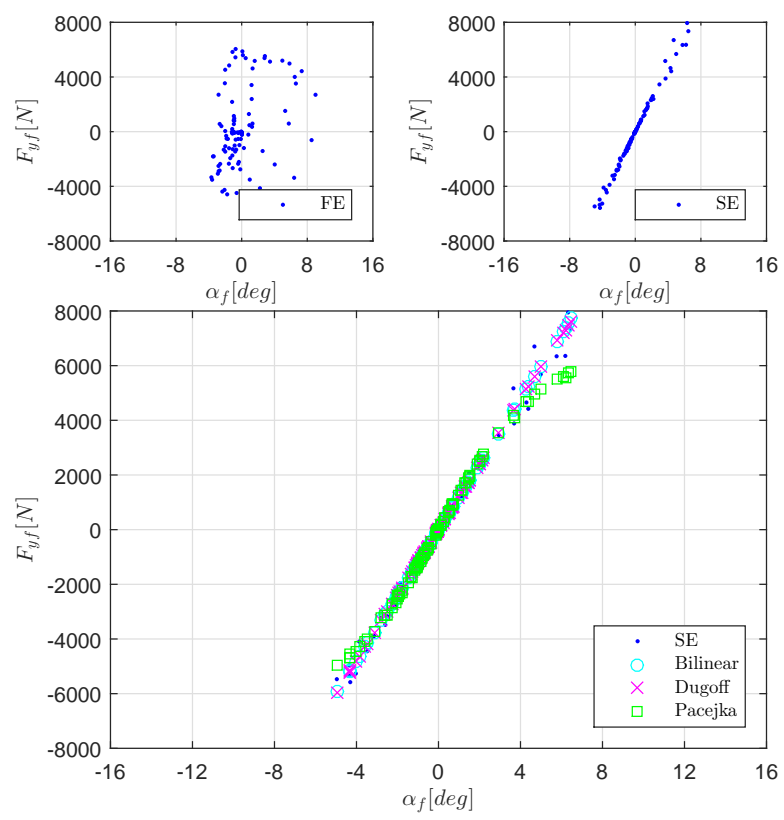

Fig. 11. Estimated and fitted tire models for front tires in double lane change.
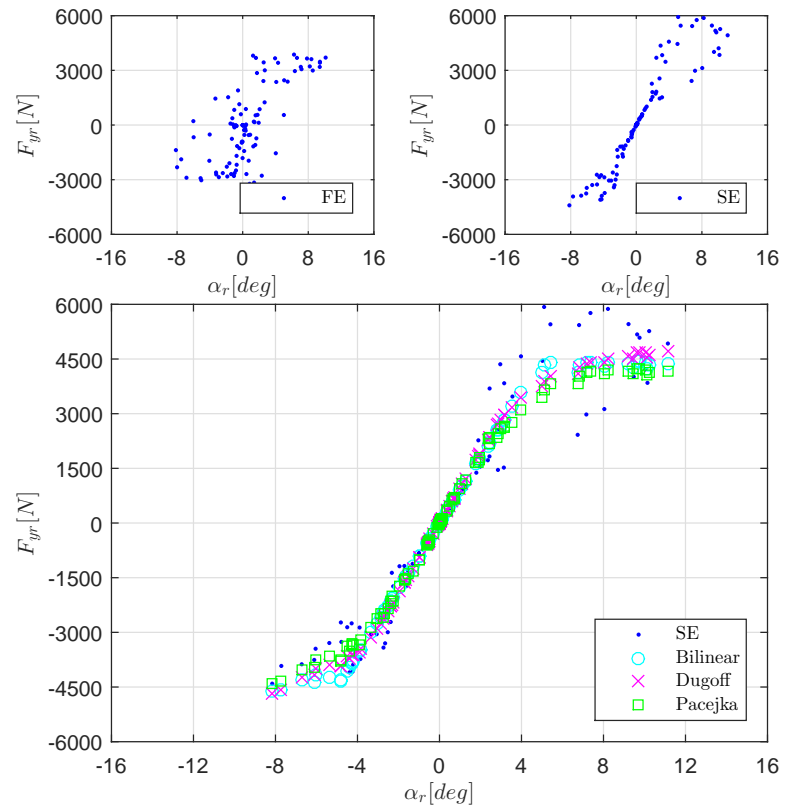

Fig. 12. Estimated and fitted tire models for rear tires in double lane change. 
TABLE II

NUMBER OF ITERATIONS REQUIRED FOR TIRE PARAMETER FITTING IN DOUBLE LANE CHANGE

\begin{tabular}{c|cc}
\hline Model & Front & Rear \\
\hline Bilinear & 3 & 3 \\
Dugoff & 5 & 4 \\
Pacejka & 20 & 7 \\
\hline
\end{tabular}

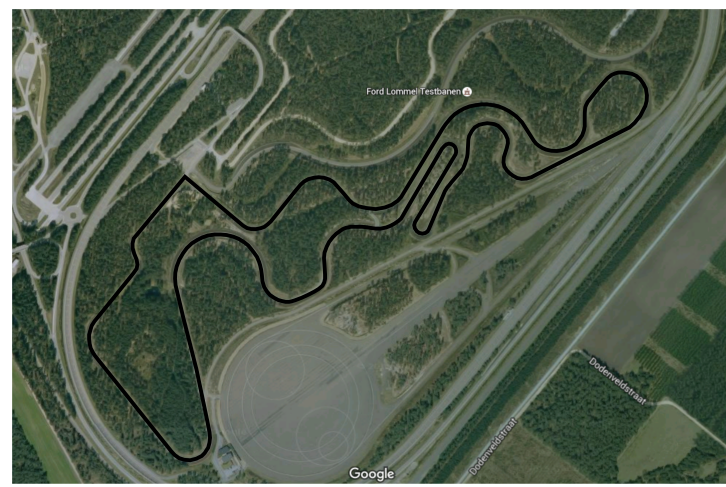

Fig. 13. The handling track at Ford Lommel Proving Ground (LPG).

ideal for testing the vehicle handling because it combines various maneuvers consisting of transient and steady state parts. Figure 13 shows a Google Earth satellite image of the track. The steering input for this track is shown in Figure 14. Figure 15 summarizes the available measurements for the estimator: the longitudinal and lateral acceleration, the four wheel speeds, and the GPS velocity. The yaw rate measurement is shown in Figure 16 together with its estimate for both estimation approaches. Figure 17 shows the estimated longitudinal and lateral velocities and compares them to the reference Corrsys Datron measurements. The sideslip angle is shown in Figure 18. Due to the low accuracy for the lateral velocity, which is caused by the low observability during straight driving, the estimated sideslip angle for the FE model provides very poor results. The SE approach however gives estimates which are very close to the measurements obtained by the Corrsys Datron system. It is also interesting to notice that the presented estimator often does not exactly track the maximum peaks of the sideslip angle. This is (partially) the result of not taking the roll and banking angle into account. Future research will focus on adding these effects. As the handling track also contains some section of straight driving,

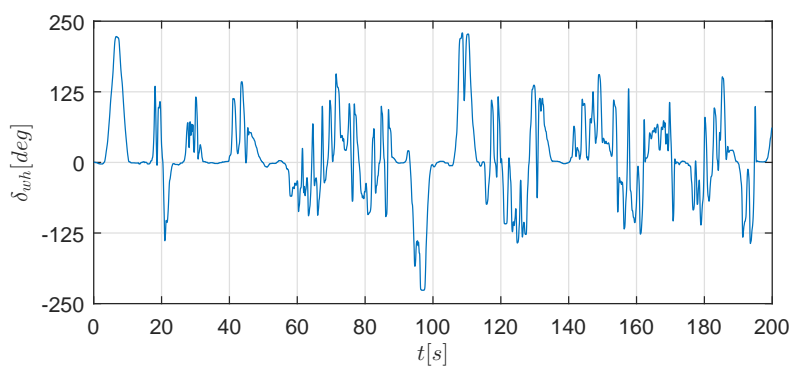

Fig. 14. Steering input for the handling track at Ford LPG.
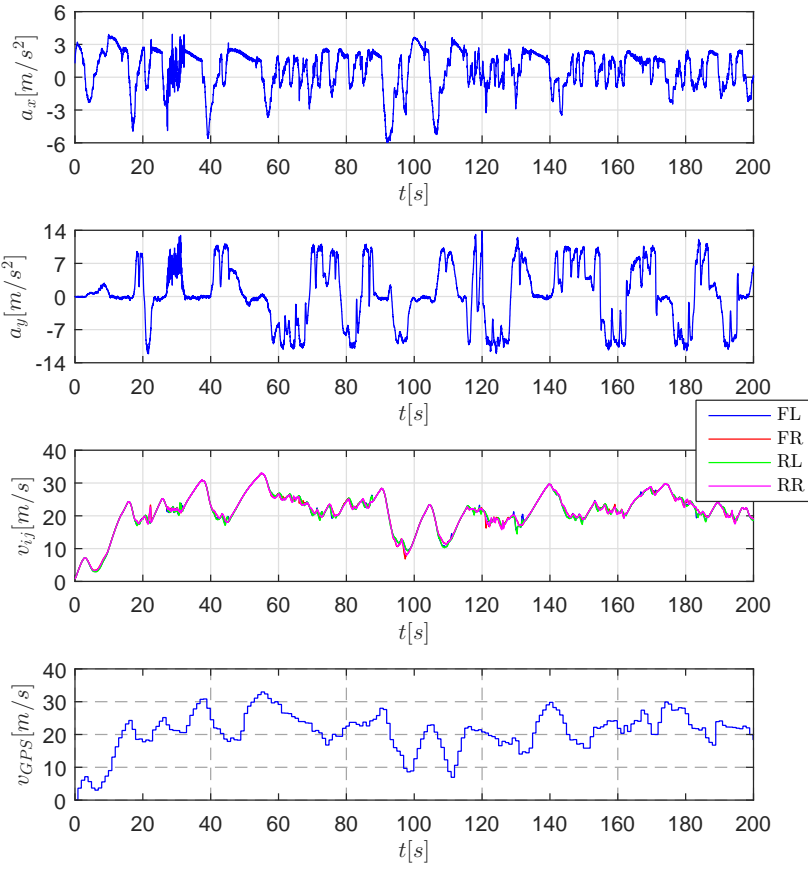

Fig. 15. Overview of the measurements for the Ford LPG handling track. Top to bottom: longitudinal acceleration, lateral acceleration, wheel speeds and GPS velocity.

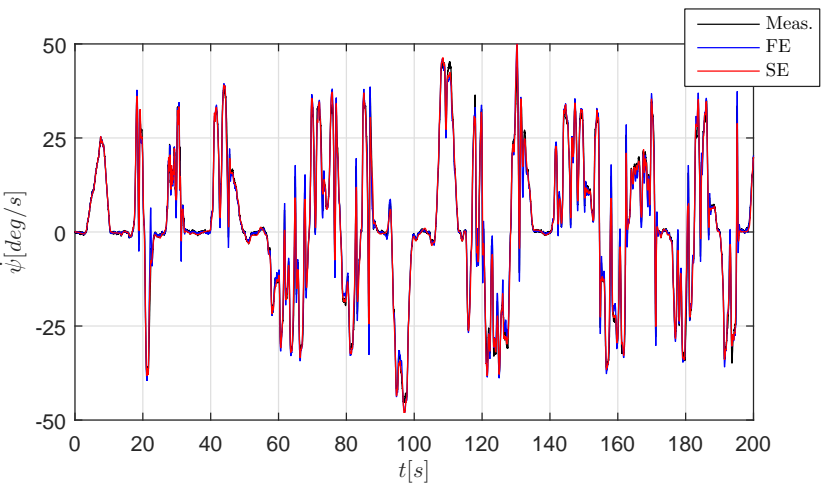

Fig. 16. The yaw rate measurement and its estimate for the Ford LPG handling track.

potential stability issues can arise for the extended Kalman filters, as discussed in Sec. III-C. It is therefor interesting to investigate the estimation covariances, shown in Figure 19. This figure clearly shows that all variances quickly reach a close to steady value. However when investigating the section of straight driving the variance of the estimated tire stiffness increases steadily when a constant model covariance is employed. During prolonged driving this will inevitably lead to an unstable estimator. The SE approach with a variable covariance, as proposed in Sec. III-C, however mitigates this issue and maintains a steady estimated variance during straight driving. For the FE approach, this instability can be seen on the variance for $v_{y}$, but here this issue cannot be mitigated as easiliy as in the case of the SE approach, such that this method 

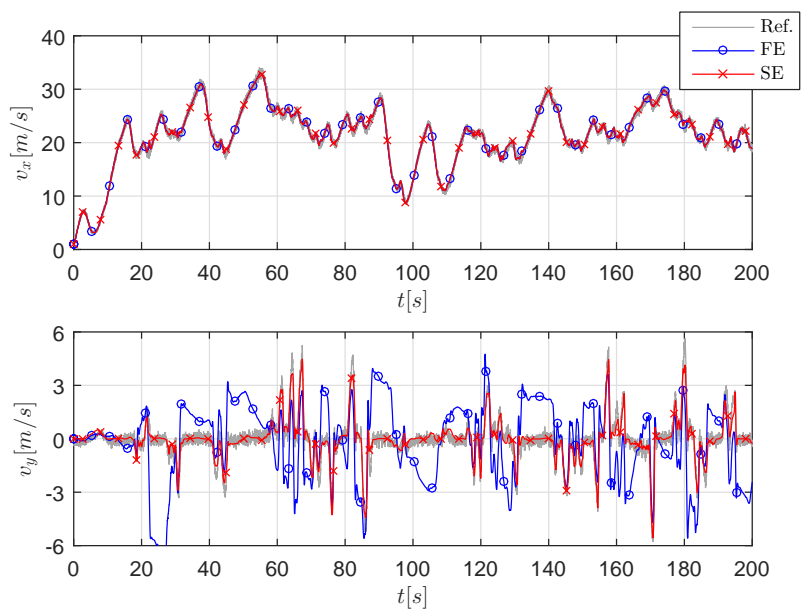

Fig. 17. The estimated longitudinal and lateral velocities for the Ford LPG handling track.

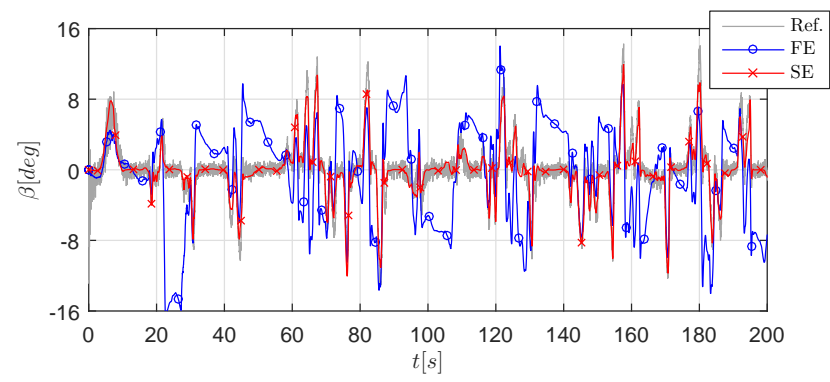

Fig. 18. The estimated sideslip angle for the Ford LPG handling track.

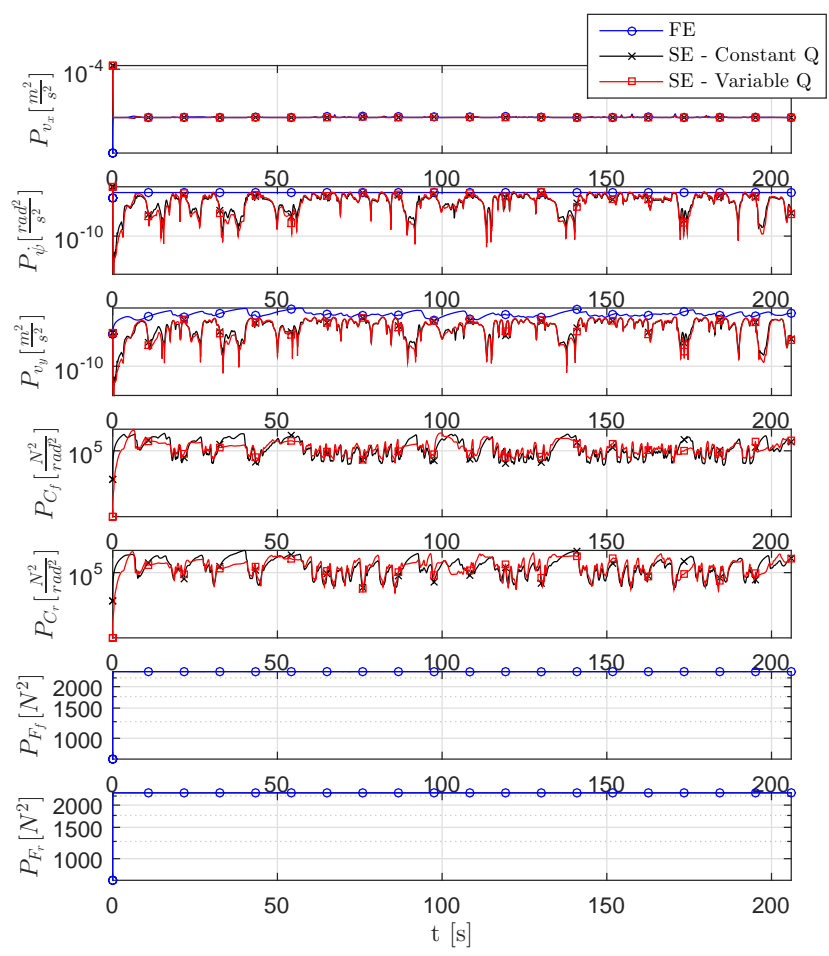

Fig. 19. Estimation variances for the FE and SE approaches on the handling track.
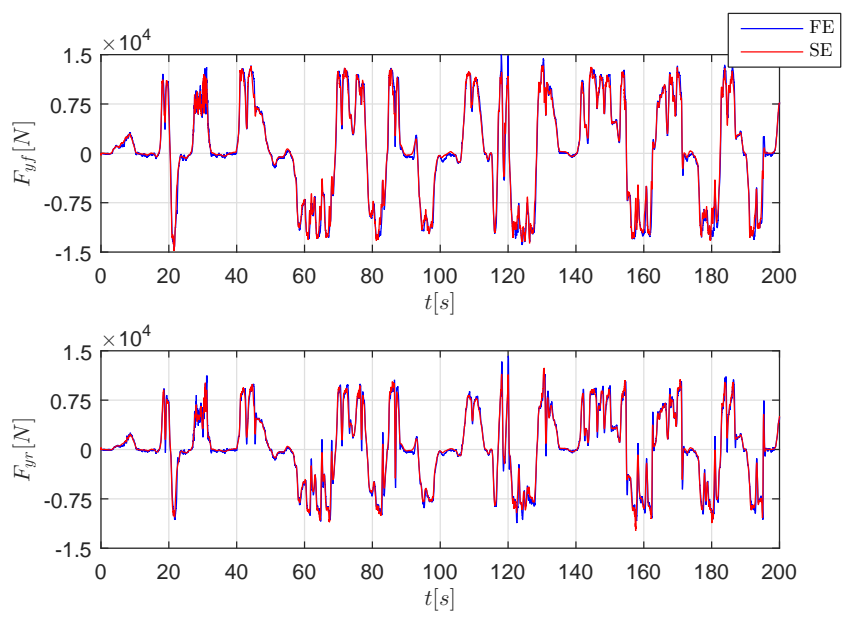

Fig. 20. Estimated lateral tire forces
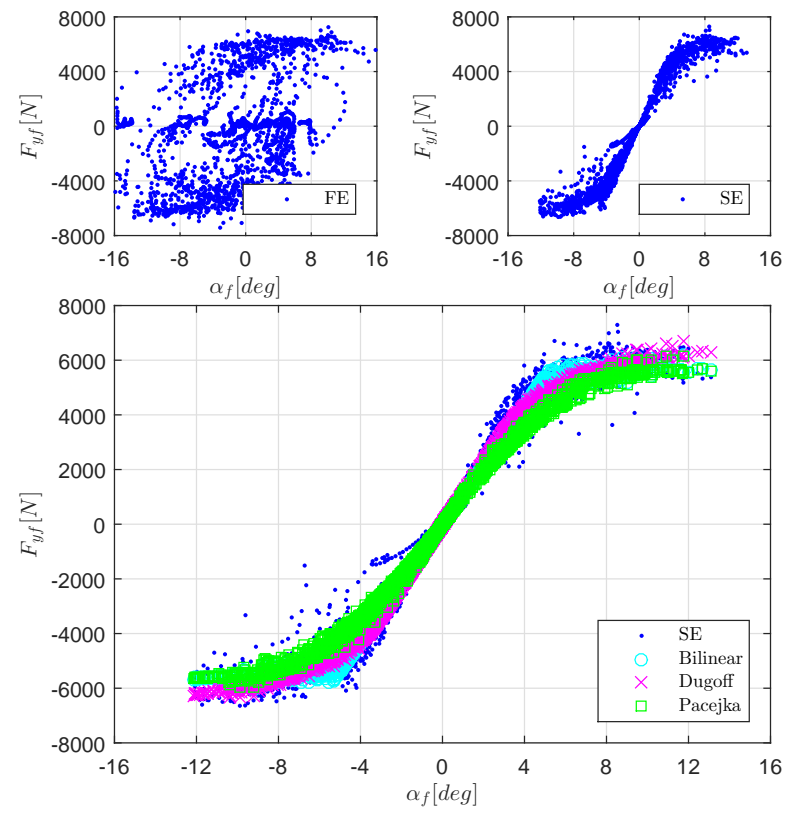

Fig. 21. Estimated and fitted tire models for front tires on handling track.

is less suitable for testing scenarios without persistent excitation. The estimated lateral tire forces as a function of time are depicted in Figure 20. For these forces the tire parameter estimation is considered next. The estimated wheel sideslip angles and the estimated forces from Figure 20 are combined in order to create a slip-force characteristic from which the tire parameters are estimated. The results are summarized in Figures 21 and 22 for respectively the front and rear tires. The plots at the top show the slip-force characteristic for the FE and SE approach. For the FE approach, it is immediately apparent that the obtained characteristic have a wide spread which makes the fitting of a valid tire model difficult. The SE approach however provides a slip-force characteristic which is very close to what is expected from a tire. The fit of the three tire models is shown in the bottom figures of Fig. 21 and 

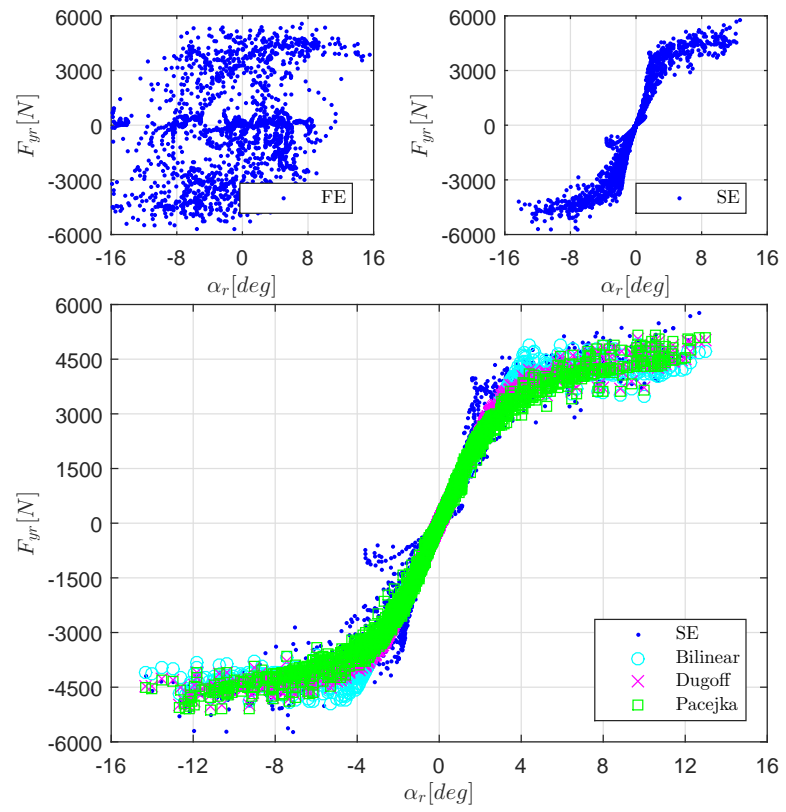

Fig. 22. Estimated and fitted tire models for rear tires on handling track.

TABLE III

NUMBER OF ITERATIONS REQUIRED FOR TIRE PARAMETER FITTING ON THE HANDLING TRACK

\begin{tabular}{c|cc}
\hline Model & Front & Rear \\
\hline Bilinear & 9 & 10 \\
Dugoff & 3 & 3 \\
Pacejka & 17 & 14 \\
\hline
\end{tabular}

Fig. 22. This fit is performed on 20000 data points and the number of iterations required for each model are summarized in Table III. Even though the current data set is very large already, fast convergence is obtained due to the high reliability of the force estimates from the SE Kalman filter. The bilinear model seems to have a bit more difficulties in fitting this strongly nonlinear model, but the computational load is still very low. The Dugoff model seems to provide the best tradeoff between accuracy and computational load for fitting the model. As a point of reference the parameter estimation for the Dugoff model takes 0.05 seconds on a regular laptop.

\section{CONCLUSION}

In this work we presented and compared two approaches for the sideslip and tire-parameter estimation in automotive applications. The presented approaches consist of two stages, a first real-time stage where sideslip and tire forces are estimated through an extended Kalman filter, and a second online stage where an optimal fit of a tire model is performed on the data generated by the first stage through a sequential quadratic program. The two presented approaches differ in how the Kalman filter model is implemented. The first approach performs a direct estimation of the lateral tire forces, the force estimation (FE) approach, whereas the second approach estimates a secant tire stiffness, the stiffness estimation (SE) approach.
We show that both methods suffer from observability issues (using regular vehicle sensors) during straight driving, which is a major limitation for general application of the methods. In order to allow for stable estimation, even in the case of unobservability during straight driving, we propose the use of a variable covariance method for the SE approach. However, the same technique cannot be applied directly to the FE model, which is a clear limitation of this approach. The proposed approaches are validated experimentally for a double lane-change maneuver and on a handling track. This validation shows the high reliability of the SE approach combined with the optimal tire fitting where the FE approach does not generate sufficiently accurate data to perform a good tire fit. The presented approach is capable of generating high fidelity data for active vehicle control applications where good knowledge of the vehicle state and tire parameters like the friction limit are essential.

\section{ACKNOWLEDGMENT}

This work is the result of a collaboration within the strategic research organisation Flanders Make. The work benefits from the Belgian Programme on Interuniversity Attraction Poles, initiated by the Belgian Federal Science Policy Office (DYSCO). The KU Leuven Research Fund is gratefully acknowledged for its support. The AIO Flanders is also gratefully acknowledged for their support within the Flanders Make MBSE4Mechatronics and GPoS-Positioning projects. The research of Frank Naets is financed by a post-doctoral grant of the Research Foundation Flanders (FWO).

\section{REFERENCES}

[1] Bosch Automotive Handbook, 9th ed. Robert Bosch, 2014

[2] "Kistler, measuring systems and sensors," www.kistler.com.

[3] H. B. Pacejka, Tire and Vehicle Dynamics, 2nd ed. SAE, Inc., 2006.

[4] Tass, "Tyre testing," https://www.tassinternational.com/tyre-testing.

[5] I. Senger and W. Kortum, "Investigations on state observers for the lateral dynamics of four wheel steered vehicles," The Dynamics of Vehicles on Roads and on Tracks, Supplement to Vehicle Systems Dynamics, vol. 18, pp. 515-527, 1989.

[6] J. Farrelly and P. Wellstead, "Estimation of vehicle lateral velocity," Control Applications, 1996., Proceedings of the 1996 IEEE International Conference on, pp. 552-557, 1996.

[7] J. Ryu, "State and parameter estimation for vehicle dynamics control using gps," Ph.D. dissertation, Standford University, December 2004.

[8] D. M. Bevly, M. Daily, and W. Travis, "Estimation of critical tire parameters using gps based sideslip measurements," in SAE Automotive Dynamics, Stability \& Controls Conference and Exhibition, February 2006.

[9] P. Yih and J. Gerdes, "Steer-by-wire for vehicle state estimation and control," in Proceedings of the International Symposium on Advanced Vehicle Control (AVEC), 2004.

[10] L. Ray, "Nonlinear state and tire force estimation for advanced vehicle control," IEEE Transactions on Control Systems Technology, vol. 3, no. 1, pp. 117-124, 1995.

[11] _ "Nonlinear tire force estimation and road friction identification: simulation and experiments," Automatica, vol. 33, no. 10, pp. 18191833, 1997.

[12] R. Anderson and D. M. Bevly, "Estimation of slip angles using a model based estimator and gps," American Control Conference, 2004. Proceedings of the 2004, vol. 3, pp. 2122-2127, 2004.

[13] _ _ "Estimation of tire cornering stiffness using gps to improve model based estimation of vehicle states," Intelligent Vehicles Symposium, 2005. Proceedings. IEEE, pp. 801-806, 2005.

[14] P. Siegrist and P. Mcaree, "Tyre-force estimation by kalman inverse filtering: applications to off-highway mining trucks," Vehicle system dynamics, vol. 44, no. 12, pp. 921-937, 2006. 
[15] H. Grip, L. Imsland, T. Johansen, T. Fossen, J. Kalkkuhl, and A. Suissa, "Nonlinear vehicle side-slip estimation with friction adaptation," Automatica, vol. 44, no. 3, pp. 611-622, 2008.

[16] G. Baffet, A. Charara, and J. Stéphant, "Sideslip angle, lateral tire force and road friction estimation in simulations and experiments," Computer Aided Control System Design, 2006 IEEE International Conference on Control Applications, 2006 IEEE International Symposium on Intelligent Control, 2006 IEEE, pp. 903-908, 2006.

[17] A. L. D. Baffet, G. ; Charara, "Estimation of vehicle sideslip, tire force and wheel cornering stiffness," Control Engineering Practice, vol. 17, no. 11 , pp. 125-1264, 2009.

[18] J.-H. Yoon and H. Peng, "A cost-effective sideslip estimation method using velocity measurements from two gps receivers," IEEE Transactions on Vehicular Technology, vol. 63, no. 6, pp. 2589-2599, 2014.

[19] X. Jin and G. Yin, "Estimation of lateral tire-road forces and sideslip angle for electric vehicles using interacting multiple model filter approach," Journal of the Franklin Institute, vol. 352, no. 2, pp. 686-707, 2015.

[20] M. U. Cuma and T. Koroglu, "A comprehensive review on estimation strategies used in hybrid and battery electric vehicles," Renewable and Sustainable Energy Reviews, vol. 42, pp. 517-531, 2015.

[21] T. R. Botha, P. S. Els, B. Jacobson, and A. Albinsson, "Vehicle motion measurements using front facing camera and digital image correlation," in ASME 2016 International Design Engineering Technical Conferences and Computers and Information in Engineering Conference. American Society of Mechanical Engineers, 2016, pp. V003T01A008V003T01A008.

[22] X. Li, X. Song, and C. Chan, "Reliable vehicle sideslip angle fusion estimation using low-cost sensors," Measurement, vol. 51, pp. 241-258, 2014.

[23] I. Davoodabadi, A. A. Ramezani, M. Mahmoodi-k, and P. Ahmadizadeh, "Identification of tire forces using dual unscented kalman filter algorithm," Nonlinear Dynamics, vol. 78, no. 3, pp. 1907-1919, 2014.

[24] S. Lee, K. Nakano, and M. Ohori, "On-board identification of tyre cornering stiffness using dual kalman filter and gps," Vehicle System Dynamics, vol. 53, no. 4, pp. 437-448, 2015.

[25] D. Simon, Optimal State Estimation: Kalman, $H_{\infty}$ and nonlinear approaches. New York: J. Wiley and Sons, 2006.

[26] Z. Lei, L. Yu, P. Ning, L. Xiaoxue, and S. Jian, "Vehicle direct yaw moment control based on tire cornering stiffness estimation," in ASME 2014 Dynamic Systems and Control Conference. American Society of Mechanical Engineers, 2014, pp. V003T49A004-V003T49A004.

[27] M. Segel, "Theoretical prediction and experimental substantiation of the response of the automobile to steering control," in Proceedings of the Institution of Mechanical Engineers: Automobile Division, vol. 10, no. 1 , January 1956, pp. 310-330.

[28] dSPACE, http://www.dspace.com

[29] R. Hermann and A. Krener, "Nonlinear controllability and observability," IEEE Transactions on Automatic Control, vol. 22, no. 5, pp. 728-740, 1977.

[30] A. Krener, "The convergence of the extended kalman filter," in Directions in Mathematical Systems Theory and Optimization, ser. Lecture Notes in Control and Information Sciences, A. Rantzer and Byrnes, Eds. Springer Berlin Heidelberg, 2003, vol. 286, pp. 173-182. [Online]. Available: http://dx.doi.org/10.1007/3-540-36106-5_12

[31] S. Ghosh and J. Rosenthal, "A generalized popov-belevitch-hautus test of observability," IEEE transactions on automatic control, vol. 40, no. 1 , 1995.

[32] K.-W. Song, J.-W. Rhim, and S.-J. Lee, "Analysis of riccati equations for the linear system with unobservable biases and its application on the ins," Control Conference, 2004. 5th Asian, vol. 3, pp. 1701-1706, 2004.

[33] R. K. Mehra, "On the identification of variances and adaptive kalman filtering," Automatic Control, IEEE Transactions on, vol. 15, no. 2, pp. 175-184, 1970.

[34] H. Dugoff, P. Fancher, and L. Segel, "Tire performance characteristics affecting vehicle response to steering and braking control inputs," Highway Safety Research Institute, Ann Arbor, Michigan, Tech. Rep., 1969.

[35] R. Guntur and S. Sankar, "A friction circle concept for dugoff's tyre friction model," International Journal of Vehicle Design, vol. 1, no. 4, pp. 373-377, 1980.

[36] H. B. Pacejka and E. Bakker, "The magic formula model," Vehicle System Dynamics: International Journal of Vehicle Mechanics and Mobility, vol. 21, pp. 1-18, 1992.

[37] T. Lefebvre*, H. Bruyninckx, and J. De Schutter, "Kalman filters for non-linear systems: a comparison of performance," International journal of Control, vol. 77, no. 7, pp. 639-653, 2004.
[38] P. E. Gill and W. Murray, "Algorithms for the solution of the nonlinear least-squares problem," SIAM Journal on Numerical Analysis, vol. 15 no. 5, pp. 977-992, 1978.

[39] SBG Systems, https://www.sbg-systems.com/products/ig500-oeminertial-systems.

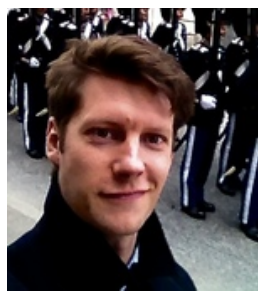

Frank Naets was born in Leuven, Belgium in 1986. He received the M.Sc. degree (2009) and a Ph.D. degree (2013) in Mechanical Engineering from KU Leuven, Belgium. Since 2009, he has been with the Noise \& Vibration research group at KU Leuven, where he is currently working as a post-doctoral researcher (FWO Fellow). Dr. Naets is an active member of Flanders Manufacturing Industry. His main research interests are in the fields of dynamic mechanical modelling, model order reduction and model based virtual sensing. His related efforts focus on dynamic mechatronic systems and vehicle dynamics.

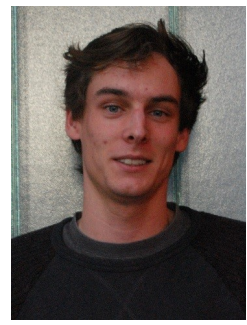

Sebastiaan van Aalst received the M.Sc. degree in mechanical engineering from the Katholieke Universiteit (K.U.) Leuven, Leuven, Belgium, in 2013. $\mathrm{He}$ is currently working as a research engineer at Flanders Make, the strategic research center for the manufacturing industry in Flanders, Belgium, and is also pursuing a Ph.D. degree at the Katholieke Universiteit (K.U.) Leuven. His research interests are in the areas of estimation and control for vehicle dynamics, multibody dynamics, and intelligent vehicles.

Boulaid Boulkroune received the M.Sc. degree in automatic control from the National Polytechnic Institute of Lorraine, France, in 2004 and the Ph.D. degree in automatic control from Henri Poincar Nancy I University, France, in 2008. He was a postdoctoral researcher at the Department of Control Engineering and System Analysis, ULB, Brussels, Belgium, till 2011. He also worked as a postdoctoral researcher at HEI, Lille and University of Picardy Jules Verne, Amiens, till 2014. Currently, He is working as a research engineer at Flanders Make in Belgium, the strategic research center for the manufacturing industry in Flanders, Belgium. His research interests are in the areas of process control, diesel engines, fault diagnosis, state estimation, fault detection and isolation, fault tolerant control.

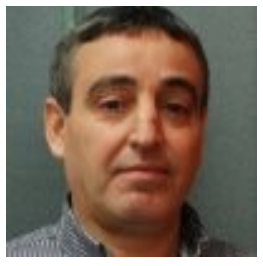

Norddin El Ghouti MSEE and Ph.D. in EEE is specialized in modeling, signal processing, and control. He is working as a project manager of several projects and leading the autonomous vehicle team at Flanders Make. He is an expert in intelligent transportation systems with focus on cooperative systems and automated/autonomous driving technologies. $\mathrm{He}$ previously worked at Aalborg University, Siemens, Holst Centre (IMEC-NL), DTI, SAM, TU/e, DAF Trucks and Flanders DRIVE.

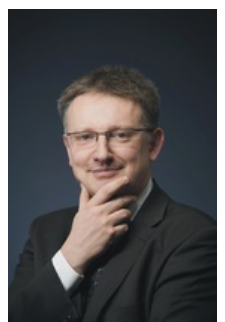

Wim Desmet was born in Waregem, Belgium in 1969. He obtained the M.Sc. degree (1992) and a Ph.D. degree (1998) in mechanical engineering from KU Leuven, Belgium. He is a Full Professor in dynamics and mechatronics at the KU Leuven Department of Mechanical Engineering, where he is currently the head of the Noise \& Vibration Research group. His research interests include virtual prototyping and experimental techniques in vibroacooustics, structural dynamics, aero-acoustics and (flexible) multibody dynamics. He has published over 220 paper in international peer-reviewed journals. Prof. Desmet is the Director of the Mechatronics \& Design Methods Department of Flanders Make, the Strategic Research Centre for the Manufacturing Industry. He is the Chairman of the KU Leuven Industrial Research Fund, General Chairman of the biennial ISMA-Noise and Vibration Engineering conference in Leuven, Belgium, and a member of the General Assembly of EARPA-European Automotive Research Partners Association, and representative for KU Leuven in EGVIA-European Green Vehicles Initiative Association. 